studied by Hertz, are fulfilled, this unclamping is sudden enough to enable oscillations to be produced.

The apparatus, called an "exciter," produces currents which change their direction from $100,000,000$ to $1, \infty 00, \infty 00,000$ times per second. Because of this extreme frequency they can produce inductive effects at a great distance. In order to render these effects simple, another electric pendulum, called a "resonator," is employed. In this new pendulum, the cut and the coil, which only serve for the unclamping, are suppressed; the two conductors reduce themselves to two very small spheres, and the wire is bent back in a circle in a way to approach the spheres to each other.

The induction due to the exciter will put this resonator in vibration the more easily as the periods of the two are less different. At certain phases of the vibration, the difference of potential of the two spheres will be large enough to produce sparks.

\section{Production of Interferences.}

IVe have thus an instrument which shows the effects of an inductive wave emitted from the exciter. We can study what happens in two ways: either expose the resonator to the direct induction of the exciter at a great distance, or else make this induction work at a short distance on a long conducting wire, along which the electric wave will go, and which will work in its turn by induction at a short distance on the resonator.

Whether the wave propagates itself along a wire or across the air, one can produce interferences by reflection. In the first case, it will reflect itself at the extremity of the wire, which it will follow again in an inverse direction; in the second, it will refiect itself on a metallic leaf which acts as a mirror. In the two cases the reflected wave will interfere with the direct wave, and we can find places where the spark of the resonator will cease to pass.

The experiments made with the long wire are easier; they furnish us with very precious instruction, but they will not serve as experimenta crucis; for in the old as well as the modern theory, the quickness of an electric wave along a wire must be equal to that of light. The experiments on the direct induction at a great distance are, on the contrary, decisive. They show that not only the quickness of propagation of induction across the air is finite, but that it is equal to the quickness of the wave propagated along a wire, complying with the ideas of Maxwell.

\section{Synthesis of Light.}

I shall insist less on other experiments of Hertz, more brilliant, but less instructive. Concentrating with a parabolic mirror the wave of induction taken from the exciter, the German savant obtains a veritable cluster of electric rays, capable of reflecting and refracting themselves regularly. The rays, if the period, already so small, were a million times shorter still would not differ from the luminous rays. We know that the sun gives out several kinds of radiation, some luminous because they act on the retina, others obscure ultra-violet or infrared, which manifest themselves by their chemical or calorific effects. The first only owe their qualities, which make them appear to us of a different nature, to a kind of physiological chance. To the physicist the infra-red does not differ more from the red, than the red from the green; the length of a wave is only greater; those of the hertzian radiations are much greater still, but there are only differences of degree, and one may say, if Maxwell's theories are true, that the illustrious Professor of Bonn has realised a veritable synthesis of light.

\section{Conclusions.}

But our admiration for so much unhoped-for success must not make us forget the progress which still remains to be accomplished. Let us therefore try to exactly summarise the results which are definitely attained.

First, the velocity of direct induction across the air is finite, without which the interferences would be impossible. The old electro-dynamics are therefore condemned. What must one put in its place? Is it Maxwell's theory (or at least something approaching it, for one would not expect the divination of the English savant to have foreseen the truth in all its details)? Although the probabilities accumulate, the complete demonstration is not yet reached.

We can measure the length of a wave of hertzian oscillations; this length is the product of the period by the velocity of propagation. We should, therefore, know this velocity if we knew the period; but this last is so small that we cannot measure it; we can only calculate it by a formula due to Lord Kelvin. This calculation leads to numbers which agree with Maxwell's theory; but the last doubts will only be done away with when the velocity of propagation has been directly measured.

This is not all: things are far from being so simple as one might think, from the above short account. Diverse circumstances come to complicate them.

First, there is round the exciter a radiation of induction; the energy of this apparatus radiates, therefore, externally, and as no fresh source comes to supply it, it soon disperses, and the oscillations die out very rapidly. It is here that one must look for the explanation of the phenomenon of multiple resonance, which was discovered by MM. Sarasin and De la Rive, and which at first appeared irreconcilable with the theory.

On the other hand, we know that light does not precisely follow the laws of geometrical optics, and the difference which produces diffraction, is more considerable as the length of the wave is greater. With the great length of the hertzian undulations these phenomena must assume an enormous importance, and trouble everything. No doubt it is fortunate, for the moment at least, that our means of observation are so coarse, otherwise the simplicity which seduced us at the first sight would give place to a labyrinth where we should be lost. It is from this probably that different anomalies arise, which have hitherto not been explained. It is also for this reason that the experiments on the refraction of rays of electric force have, as I said above, but little demonstrative worth.

There still remains a difficulty which is more serious, but which is no doubt not insurmountable. According to Maxwell, the coefficient of electrostatic induction of a transparent body ought to be equal to the square of its index of refraction. This is not so; the bodies which follow Maxwell's law are exceptions. We are evidently in the presence of phenomena much more complex than we thought at first ; but one has not been able to explain anything, and the experiments themselves are contradictory.

There still remains, therefore, much to be done; the identity of light and electricity is from to-day something more than a seducing hypothesis : it is a probable truth, but it is not as yet a proved truth.

\section{THE RECENT WORK OF THE CATARACT CONSTRUCTION COMPANY.}

SOME arrangements recently made by the Cataract Construction Company show that the works are extending in a very satisfactory manner. The Niagara Falls Paper Company is now well under way. They make paper from wood pulp, and a large amount of power is used for grinding the trees down into pulp. They have fixed turbines in their own wheel-pit, and take water from

No. I 279 , vor. 50 ] 
the Cataract Construction Company's canal, and pass it after use into the tunnels belonging to this Company. The turbines drive a vertical shaft which, at the surface of the ground, is geared by bevel wheels to the main shaft, on which are the pulp grinders. This is the only case likely to occur where the power is used directly. In all other cases it is likely to be transmitted electrically. The first electrical application is to the works of the Pittsburgh Reduction Company for the manufacture of aluminium. The Niagara Falls Power Company has undertaken to supply them with continuous current at 160 volts to the extent of 7000 horse-power. The first 1500 horse-power is now being put in. In order to do this, Prof. Forbes has arranged to carry the alternating current by two circuits in two phases. This is the character of current generated by the dynamos. At the works, 2500 feet from the powerhouse, the current is to be transformed from 2000 volts to I 5 volts alternating. It is then passed through three of the commutating machines, like those shown by Schuckert and others at the Frankfort Exhibition of IS9I. These are continuous current machines, each with four rings attached to certain bars of the commutator. The alternating current is passed to these rings by brushes; the armature revolves synchronously with the generator in the power-house and with the turbine. The other brushes, which rub on the commutator, give off continous current to the aluminium baths at 160 volts, thu E.M.F. being (in normal conditions) raised in its change form alternating to continuous current in the ratio of $\sqrt{2}:$ I. A shunt direct current with variable resistance in the circuit excites the field magnets. It is found that the electromotive force of the direct current can thus be regulated. The explanation of this remarkable fact has been worked out by Mr. Steinmetz. The whole of this plant, transformers, commutating machines, and switchboard was tendered for by different firms, and the bid of the General Electric Company has been accepted ; 33 per cent. of spare plant is held in reserve. The dynamos and power-house switch-board, on the other hand, are in the hands of the Westinghouse Company. It is expected that both types of machinery will be in operation in the course of a few months.

The patent for Prof. Forbes' construction of dynamo, which is being built, has been just allowed by the United States Patent Office. One object to be attained was to have a fixed armature, so that the armature might be wound so as to give a very high E.MI.F. without being subjected to the enormous centrifugal forces of the revolving part. Another object was to attain a maximum fly-wheel effect. with a minimum weight. The revolving parts of the turbine and dynamo, and the vertical shaft connecting them, are all supported hydraulically by means of a piston in the turbine. The supporting power of this piston limited the weight of the revolving part of the dynamo to So,ooolbs. The governor of the turbine demanded (to fulfil the required conditions of regulation) a momentum equal to that of $1,100,000,000$ lbs., moving at the rate of I foot per second.

Both of these objects are attained by Prof. Forbes' construction, which consists in making the armature fixed and ring-shaped, with a space inside for getting at the bearings, and in making the fields of a bell-shape, the poles being on the inside of a nickel steel ring, which is supported by the top piece or cover, which in its turn is rigidly fixed to the vertical axis. This novel construction gives all the fly-wheel effect required without making the weight too great. Every design which had been made previously required the addition of a flywheel costing at least $£$ Soo or $£$ rooo. The construction for which Prof. Forbes' patent has now been granted has also the great merit that the magnetic pull between the armature and the fields tends to diminish the breaking strain of centrifugal force on the revolving part to a very No. 1279 , voL. 50] sensible amount. When the revolving part is inside the magnetic pull assists centrifugal force in its destructive effect.

\section{THE EPPING FOREST CONTROVERSY.}

THE meeting of the Essex Field Club, which, as we announced last week, had been convened for the inspection of the thinned districts of Epping Forest, was in every way a remarkable gathering. Nearly 150 members and visitors assembled in Monk's Wood, among them, in addition to the conductors and officers of the Club, being Sir John Lubbock, Mr. J. Bryce, MI.P., Chancellor of the Duchy of Lancaster, Prof. Boulger, Prof. WV. R. Fisher, Dr. Church, Mr. Arthur Lister, Mr. Andrew Johnston (chairman of the Essex County Council), Mr. David Howard, Mr. Salmon (chairman of the Epping Forest Committee), Sir Frederick Young, Mr. Bernard Gibson, and others interested in the question of the Forest management. A thorough examination of Monk's Wood was made, Mr. E. N. Buxton giving a detailed explanation of the policy which the Conservators had been pursuing, and pointing out the reasons that had led to the present necessity for thinning. The party were then conducted to Lord's Bushes, which, as an example of the beautifying effect of judicious thinning, is almost unrivalled, this district having been submitted to the operation repcatedly since the Forest was taken over by the present Conservators. After tea at the Royal Forest Hotel, Chingford, a meeting of the Club was held, Mr. F. Chancellor, the President, in the chair. The discussion was opened by Prof. Meldola, who commenced by explaining that the statements which had been inserted in the newspapers respecting the attitude taken by the Club in the present controversy had been made without authority, and were devoid of foundation. He then went on to show that the observations made by him in $188_{3}$ in connection with the agitation against the railway scheme with which the Forest had then been threatened (see the article quoted in our columns in last week's note), had recently been applied to the present thinning operations without his concurrence, and in direct opposition to the views which he had formed after five visits to the districts now being dealt with, and after an intimate acquaintance with the Forest for a period of more than twenty years. Having explained the general grounds on which he based his opinions, he stated that in his belief the Forest as a whole showed a marked improvement since it had been under the care of the present management, and he considered that much of the recent criticism had been most unjust, and the newspaper accounts exaggerated to an extent bordering on the ludicrous. Two or three speakers took an adverse position, but Prof. Boulger, Mr. Howard, Mr. F. C. Gould, Mr. W. Crouch, the President, Mr. William Cole, and others who knew the Forest well, expressed general approval of the recent operations. Mr. Angus Webster, the Duke of Bedford's forester, who accompanied the party, was of course obliged to reserve his opinion owing to his official connection with the committee of experts appointed by the Corporation, the report of this committee not having as yet been presented. As the outcome of the discussion, it may safely be said that the public will not be so ready in future to give credence to the opinions of irresponsible and inexperienced scribblers who, often with the best of motives, may seriously hamper the work of the Conservators in their endeavour to restore the Forest to a more natural condition in those parts which have for so many centuries been made unnatural and unsightly by the existence of the rights of lopping. Although it had not been the original intention of the officers and conductors to allow a formal motion to be put, a strong desire was expressed that this should be done, and on 